\title{
Spectroscopy of the Crab Pulsar
}

\author{
G. M. Beskin ${ }^{1,3}$ and V. V. Neustroev ${ }^{1,2}$ \\ 1 Special Astrophysical Observatory, Nizhnij Arkhyz, Karachaevo-Cherkesia, 369167, Russia \\ 2 Department of Astronomy and Mechanics, Udmurt State University, 1, Universitetskaia, Izhevsk, 426034, Russia \\ e-mail: benj@uni.udm.ru \\ ${ }^{3}$ Isaac Newton Institute of Chile, SAO Branch, Chile
}

Received 5 April 2001 / Accepted 7 May 2001

\begin{abstract}
We present new, high signal-to-noise spectroscopy of the Crab Pulsar in the range $\lambda \lambda 5000-7000 \AA$. The observations were carried out with the 6-meter telescope of the SAO with the spectrograph SP-124 with a resolution of $2 \AA$. After reduction of the Crab Nebula emission, the dereddened pulsar spectrum can be fitted by a power law with $\alpha=-0.15 \pm 0.15$. There are no lines (emission and absorption) with a relative intensity (depth) of more than $2 \%$ (with a confidential probability of $99 \%$ ). At this level we did not find absorption at $\lambda 6000 \AA$, possibly of ion-cyclotron origin, as detected by Nasuti et al. (1996). This detail can be variable as a consequence of the variability of the ion ejection from the neutron star (NS) surface. We suggest a possible relationship between ejection intensity and inhomogeneity of the NS surface connected with pulsar glitches.
\end{abstract}

Key words. stars: pulsars: individual (Crab Pulsar); pulsars: general - stars: neutron

\section{Introduction}

The pulsar in the Crab Nebula (PSR 0531+21) was the first pulsar identified at optical wavelengths (Cocke et al. 1969). Its radiation, with a period of pulsation of $0.033 \mathrm{~s}$, was later detected at all frequencies from radio to gamma. The pulsar turned out to be the wellknown south-preceding star in the center of the nebula which emits a featureless continuum (Minkowski 1942). This property of its emission was confirmed in the time resolved spectroscopy of Oke (1969) and Lynds (1969).

It is rather curious that no spectroscopic observations of the Crab pulsar were made for over 20 years. Nasuti et al. (1996) obtained an averaged spectrum of the object that exhibited an absorption line at $6000 \AA$. This result, if confirmed, could be evidence for a radically new possibility in the study of pulsars. Data are also available on the presence of a similar feature in the spectra of Geminga as well (Mignani et al. 1998; Martin et al. 1998). Since the appearance of such a line can be explained as being of ion-cyclotron ( $\mathrm{H}$ and/or $\mathrm{He}$ ) nature, its characteristics represent the conditions in the pulsar magnetosphere (atmosphere) - the intensities of the magnetic and gravitational fields, the number densities of particles of different types and their energy and spatial distributions (Jacchia et al. 1999). On the other hand, light elements in young pulsars may appear in the atmosphere itself, as a result of "floating up" in the liquid iron crust at early stages of

Send offprint requests to: G. Beskin, e-mail: beskin@sao.ru its existence (Lai \& Salpeter 1997) or as a consequence of bombarding by energetic particles from the magnetosphere (Beskin 1999). Hence, the parameters of the ioncyclotron lines $\mathrm{H}$ and $\mathrm{He}$ (and their presence) are due to the state of the pulsar's surface and give information about it.

The principal goal of our observations was to derive an averaged spectrum of the pulsar in the Crab Nebula with a high signal-to-noise ratio around $\lambda 6000 \AA$ to check the detection of the cyclotron line. Because the greater part of the pulsar radiation comes in the primary (60\%) and secondary $(30 \%)$ pulses, we searched for the line exactly at these two phases of the period.

We have reported in brief on our work in the paper by Komarova et al. (1996). In the present paper, results of these investigations and their interpetation are presented in detail.

\section{Observations}

Observations of the Crab pulsar were carried out with the 6-m telescope of the Special Astrophysical Observatory during 4 nights, January, 13-16, 1996, using a CCD attached to the spectrograph SP-124 at the Nasmyth1 focus. The weather conditions were fine - the seeing was $1^{\prime \prime} 0-1$ ". 3 on different nights. A total of 35 spectra with a resolution of $2.1 \AA$ /pixel, covering a range of 5000-7000 $\AA$, were obtained. The exposure time on different nights ranged from $3 \mathrm{~min}$ to $10 \mathrm{~min}$, the total 
Table 1. Log of observations.

\begin{tabular}{ccccc}
\hline $\begin{array}{c}\text { Date } \\
\text { (1996 Jan.) }\end{array}$ & UT Start & $\begin{array}{c}\text { Exposure } \\
(\mathrm{sec})\end{array}$ & $\begin{array}{c}\text { Number } \\
\text { of exposures }\end{array}$ & $\begin{array}{c}\text { Wavelength } \\
\text { range }(\AA)\end{array}$ \\
\hline 11 & $22^{\mathrm{h}} 27^{\mathrm{m}} 11^{\mathrm{s}}$ & 180 & 24 & $5000-7050$ \\
12 & $23^{\mathrm{h}} 58^{\mathrm{m}} 09^{\mathrm{s}}$ & 180 & 6 & $5000-7050$ \\
14 & $00^{\mathrm{h}} 52^{\mathrm{m}} 40^{\mathrm{s}}$ & 180 & 2 & $5000-7050$ \\
16 & $19^{\mathrm{h}} 29^{\mathrm{m}} 46^{\mathrm{s}}$ & 180 & 1 & $5000-7050$ \\
16 & $19^{\mathrm{h}} 36^{\mathrm{m}} 00^{\mathrm{s}}$ & 600 & 3 & $5000-7050$ \\
\hline
\end{tabular}

observing time was $129 \mathrm{~min}$. The observations were longslit ( $40^{\prime \prime}$ long and $1^{\prime \prime} .5$ wide) with a north-east orientation of the slit. To calibrate the wavelength, the spectrum of a $\mathrm{He}-\mathrm{Ne}-\mathrm{Ar}$ lamp was used for each observing night, while to correct for the inhomogeneous spectral sensitivity of the CCD chip and to transform intensity in the flux units, spectra of the standard star PG 129b (Oke 1977) were obtained. The log of the observations is given in Table 1 .

\section{Data reduction}

The two-dimensional spectral images were processed using the system MIDAS data reduction package. The primary reduction included bias correction and division by the flat field, after which the cosmic particle traces were removed from the image. At the next stage of the processing it was necessary to correctly subtract the sky from the image. This procedure, generally simple, turned out to be an involved problem in the reduction of spectral data for the Crab pulsar. Indeed, the narrow band of the pulsar continuum emission on the two-dimensional image is crossed by a set of emission lines produced in the Crab Nebula (Fig. 1).

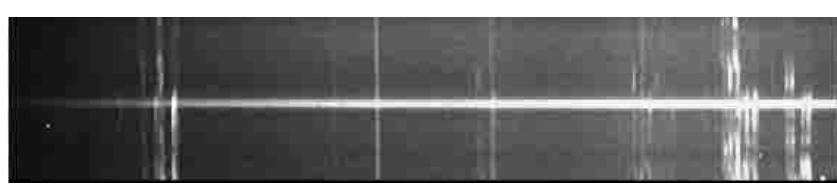

Fig. 1. Original image of the Crab pulsar spectrum. Continuous lines originate from the night sky, discontinuous ones from the Crab Nebula.

Due to the significant inhomogeneity of the Nebula, these lines are highly variable, sometimes almost disappearing entirely. The application of standard techniques to background subtraction, which achieved the highest quality final spectrum, failed to get rid of the Nebula lines. This is why, in the processing, the sky background on the two-dimensional spectral image was determined in the immediate neighbourhood of the pulsar spectrum. This permitted us to expect, taking into account the high quality of the spectra and favourable observing conditions, constant line intensities of the Nebula within at least a few pixels and, therefore, a more correct subtraction of the background. As a result we actually got rid of many Nebula lines, although the strongest of them still remained.
The final stage of the reduction was the correction for interstellar extinction. We used the extinction coefficients in different parts of the spectrum determined by Persival et al. (1993), assuming that $E_{B-V}=A_{v} / 3.1=0.51$ and $A_{\lambda} / E_{B-V}=-7.51 \log \left(\lambda / \lambda_{0}\right)+1.15 ; \lambda_{0}=1 \mu \mathrm{m}$, that reflects galactic extinction in the range $4800 \AA \div 10000 \AA$ (Savage \& Mathis 1979).

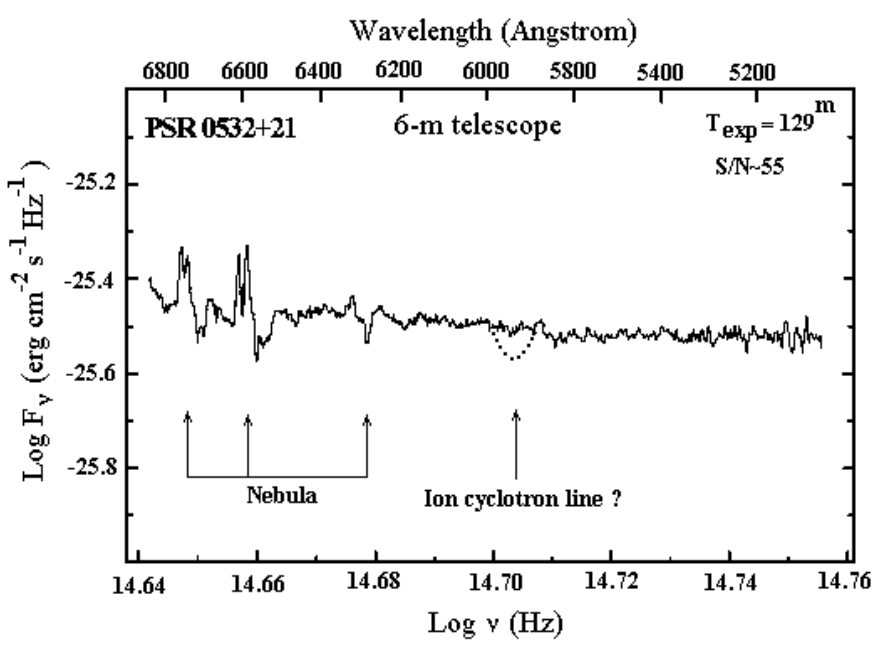

Fig. 2. Spectrum of the Crab pulsar.

\section{Results and discussion}

The total flux-calibrated extinction corrected spectrum of the Crab pulsar is displayed in Fig. 2. The spectrum is characterized by the continuum following a power law with $\alpha=-0.15 \pm 0.15$. It does not contain any other features except the rest of the Crab Nebula emission lines. The broad absorption feature revealed by Nasuti et al. (1996), which is indicated in our figure on the same scale, is also absent. We have also checked all individual and daily total spectra. None of them shows significant absorption features. The noise of the spectrum gives a $2 \%$ upper limit for relative intensity (depth) of any lines at a significant level of $<1 \%$.

Thus, we could reliably detect lines even 5-7 times weaker than the detail at $\lambda 5920 \AA$ with a width of $100 \AA$ and depth of 0.15 (Nasuti et al. 1996). The absence of this feature is also noted by Sollerman et al. (2000) and Carramiñana et al. (2000). Is the result of Nasuti et al. then wrong? The authors themselves note this possibility. Indeed, the spectrum of the Crab pulsar is most likely still to be purely continuous. However, an alternative cannot be completely rejected, namely time variations of the line depth, which results in it either being detected or not.

The reason for analysing this possibility is the probable detection of the ion-cyclotron line in Geminga (Mignani et al. 1999). Hydrogen ions (and, possibly, helium ions) can appear in the atmosphere of pulsars through accretion of the interstellar plasma (Lai \& Salpeter 1997). By virtue of the young age of the Crab pulsar, the powerful 
Table 2. Parameters of the glitches close to the Crab pulsar spectral observations.

\begin{tabular}{|c|c|c|c|c|c|c|c|}
\hline \multicolumn{5}{|c|}{ Glitch } & \multicolumn{3}{|c|}{ Spectroscopy } \\
\hline Number & $\begin{array}{c}\Delta \nu \\
\left(10^{-7} \mathrm{~Hz}\right)\end{array}$ & $n^{*}$ & $\begin{array}{c}\tau_{n} \\
\text { (days) }\end{array}$ & $\overline{\text { Data }}$ & Data & $\begin{array}{c}\text { Line } \\
\text { detection }\end{array}$ & Paper \\
\hline \multirow[t]{3}{*}{5} & 18.5 & 1 & 18 & $89-08-29$ & \multirow{4}{*}{$91-01$} & \multirow{4}{*}{ Yes } & \multirow{4}{*}{ Nasuti et al. (1996) } \\
\hline & & 2 & 265 & & & & \\
\hline & & & & & & & \\
\hline 6 & 3 & 1 & 2.6 & $92-11-21$ & & & \\
\hline 7 & 0.8 & 1 & 3.2 & $95-10-30$ & \multirow{3}{*}{$96-01-13,15$} & \multirow{3}{*}{ No } & \multirow{3}{*}{ This paper } \\
\hline & & & & & & & \\
\hline 8 & 6.6 & 1 & 10.3 & $96-06-25$ & & & \\
\hline \multirow[t]{2}{*}{11} & \multirow[t]{2}{*}{2.6} & \multirow[t]{2}{*}{1} & \multirow[t]{2}{*}{2.9} & \multirow[t]{2}{*}{$97-12-30$} & & & \\
\hline & & & & & $98-12-13,14$ & No & Carramiñana et al. (2000) \\
\hline \multirow{3}{*}{12} & \multirow{3}{*}{2.9} & \multirow{3}{*}{1} & \multirow{3}{*}{3.4} & \multirow{3}{*}{$99-10-01$} & $98-12-24,25$ & No & Sollerman et al. (2000) \\
\hline & & & & & & & \\
\hline & & & & & $99-10-18,20$ & No & Carramiñana et al. (2000) \\
\hline
\end{tabular}

* 1 - Short term transient component.

2 - Long term transient component.

relativistic wind and the radiation pressure prevent the penetration of interstellar matter through the light cylinder surface. Nevertheless, in its crust, there may be present the ions of light elements either of relic origin, which have remained there since the early stage of existence of the neutron star (Lai \& Salpeter 1997), or which have appeared as a result of interaction between iron and fast particles filling the magnetosphere (Beskin 1999). A certain portion of these ions get into the atmosphere surrounding the neutron star. It will be emphasized that the number of ions and, therefore, the cyclotron line intensity, depends on the state of the pulsar's surface. This is due to the dependence of the work surface function on the local intensities of the magnetic and induced electric fields i.e. on the degree of roughness of the outer crust. In turn, the latter can essentially change its structure as a result of interaction with the superfluid liquid in the inner regions of the pulsar, which are reconstructed spontaneously from time to time (Ruderman 1991; Allen \& Horvath 1997; Baykal et al. 1999). These transformations are likely to cause both the famous "glitches" (occasional interruptions of the spin-down by sudden period discontinuities) and the "timing noise" (irregularities in the rotation rate), which have long been observed in the radio range (Boynton et al. 1972; Groth 1975). It can thus be assumed that the enhancement of the number density of hydrogen and/or helium ions may be correlated with glitches and timing noise. The ion-cyclotron lines may therefore appear near the moments of glitches (before and after them), as well as in the periods of increased intensity of the timing noise.

We have compared the moments of spectral investigations of the Crab pulsar and glitches recorded. They are listed in Table 2 together with their basic parameters - the jump in the pulsar rotational frequency $(\Delta \nu)$ and the characteristic times of relaxation of rapid $\left(\tau_{1}\right)$ and slow $\left(\tau_{2}\right)$ transient jump components which have been derived from the observations and modeling of Lyne et al. (1993) and Wong et al. (2001). It is seen that in a certain sense the observations of Nasuti et al. (1996) are, indeed, unique. They were made 1.5 years after the most powerful glitch ever recorded in the pulsar. The amplitude of the frequency jump, $18 \times 10^{-7} \mathrm{~Hz}$, is 6 times as high as this parameter in other glitches. The rapid relaxation continued also 6 times longer than in other cases. The slow component of the frequency relaxation, which was lacking in other glitches, has a characteristic decay time of 265 days. In other words, this glitch was associated with the longest and most intense transformation of the neutron star parameters. Its consequences might have appeared 1.5 years later during the observations of Nasuti et al. (1996). The observations of Carramiñana et al. (2000) were conducted 18 days after the glitch in October 1999, but here the frequency jump and the relaxation time were close to a minimum.

Thus, one cannot exclude the possibility that the ioncyclotron line in the spectrum of the Crab pulsar is real. Its appearance may be due to ejection of a sufficient number of ions from the neutron star surface, when it was, transformed during and after the giant glitch of 1989. Spectral monitoring of the Crab pulsar nebula coordinated with its radio timing can allow one to confirm or argue against this effect.

Acknowledgements. This investigation was supported by the Russian Ministry of Science, Russian Foundation of Basic Researches (grants 98-02-17570, 01-02-17857), Federal Program "Astronomy" and INTAS (grant 96-0542).

We thank T. Tupolova and V. Debur for assistance in the preparation of this paper. 


\section{References}

Allen, M. P., \& Horvath, J. E. 1997, ApJ, 488, 409

Baykal, A., Alpar, M. A., Boynton, P. E., \& Deeter, J. E. 1999, MNRAS, 306, 207

Beskin, V. 1999, UFN, 169, 1169

Boynton, P. E., Groth, E. J., Hutchingson, D. P., et al. 1972, ApJ, 175, 217

Carramiñana, A., C̆adež, A., \& Zwitter, T. 2000, ApJ, 542, 974

Cocke, W. J., Disney, M. J., \& Taylor, D. J. 1969, Nature, 221, 525

Groth, E. J. 1975, ApJS, 29, 443

Jacchia, A., De Luca, F., Lazzaro, E., et al. 1999, A\&A, 347, 494

Komarova, V. N., Beskin, G. M., Neustroev, V. V., et al. 1996, J. Kor. Astr. Soc., 29, 217

Lai, D., \& Salpeter, E. E. 1997, ApJ, 491, 270

Lynds, K. 1969, ApJ, 157, L11
Lyne, A. G., Pritchard, R. S., \& Smith, F. G. 1993, MNRAS, 265, 1003

Martin, C., Halpern, J. P., \& Schiminovich, D. 1998, ApJ, 494, 211

Mignani, R., Caraveo, P. A., \& Bignami, G. F. 1998, A\&A, 332, L37

Minkowski, R. 1992, ApJ, 96, 199

Nasuti, F. P., Mignani, R., Caraveo, P. A., \& Bignami, G. F. 1996, A\&A, 314, 849

Oke, J. B. 1969, ApJ, 156, L49

Oke, J. B. 1977, AJ, 99, 1621

Percival, J. W. 1993, ApJ, 407, 276

Ruderman, M. 1991, ApJ, 382, 587

Savage, B. D., \& Mathis, J. S. 1979, ARA\&A, 17, 73

Sollerman, J., Lundqvist, P., Lindler, D., et al. 2000, ApJ, 537, 861

Wong, T., Backer, D. C., \& Lyne, A. G. 2001, ApJ, 548, 447 